\title{
A newly established culture method highlights regulatory roles of retinoic acid on morphogenesis and calcification of mammalian limb cartilage
}

\author{
Eizo Masuda ${ }^{1}$, Kota Shirai ${ }^{1}$, Kenji Maekubo ${ }^{1}$ and Yohei Hirai ${ }^{1,2}$ \\ ${ }^{1}$ Department of Bioscience, Kwansei Gakuin University, Sanda, Japan and ${ }^{2}$ Research \\ Center for Intelligent Bio-Materials, Kwansei Gakuin University, Sanda, Japan
}

BioTechniques 58:318-324 (June 2015) doi 10.2144/000114300

Keywords: chondrogenesis; retinoic acid; three dimensional culture; limb; cartilage; calcification; morphogenesis

Supplementary material for this article is available at www.BioTechniques.com/article/114300.

During mammalian embryogenesis, sclerotome-derived chondrocytes in the limb bud are arranged into a complicated bone shape with specific areas undergoing hypertrophy and calcification, creating a regionspecific mineralized pattern in the cartilage. To follow chondrogenesis progression in vitro, we isolated limb cartilage from mice on embryonic day 13 (E13) and cultured it at the air-liquid interface after microsurgical removal of the ectoderm/epidermis. Explants underwent proper morphogenesis, giving rise to complete templates for limb bones in vitro. We found that region-specific calcification patterns resembling limbs of prepartum mature embryos could be induced in explants using culture medium containing high concentrations of $\mathrm{CaCl}_{2}(\mathrm{Ca})$, ascorbic acid (AA), and $\beta$-glycerophosphoric acid (BGP). In this culture system, excess amounts of all-trans retinoic acid (RA) severely disrupted morphogenesis and calcification patterns in limb cartilage. These effects were more pronounced in forearms than in phalanges. Although dissociated, the nascent chondrocytes in culture did not give rise to cartilage units even though augmented calcification was induced in these cell aggregates in the presence of RA. Taken together, our newly established culture system revealed that RA independently regulates three-dimensional morphogenesis and calcification.

During limb osteogenesis in the mammalian embryo, chondrogenesis precedes angiogenesis and endochondral ossification by osteogenic cells carried through the bloodstream. In chondrogenesis, sclerotome-derived chondroblasts form a distinct arrangement, followed by regional hypertrophy and calcification in the nascent cartilage $(1,2)$. The precise control of morphogenesis and spatiotemporal differentiation of the developing cartilage predetermine the shape and physical properties of mature bone (3). Osteogenesis in this intricately shaped body part is orchestrated by chondrocyte behaviors in specific cartilaginous units connected to each other to form a complex framework. For example, chondrocytes in the zeugopod form complete parallel templates for ulna and radius, with gradual calcification expanding from their central portions, while chondrocytes in the autopod construct tandem chains that create the carpal and phalangeal cartilages, with active calcification starting at the tips between them (4).

Together with signals propagated by other factors, including bone morphogenetic proteins (BMPs), fibroblast growth factor (FGF), sonic hedgehog (Shh), and Wnt, all-trans retinoic acid (RA) signaling plays a vital role in the formation of limb bone during mammalian embryogenesis as well as amphibian regeneration (5-7).
RA binds to dimeric receptor complexes consisting of $R A$ receptors (RARs) and retinoid $X$ receptors ( $R X R s$ ) and directly affects transcription of several factors that carry positional information, such as the Hox genes (8-10). To determine the precise role of $\mathrm{RA}$, transgenic animals with mutations in the RA signaling pathway were generated, and chondrogenic defects in these animals indicated a function for RA in the development of mammalian limbs $(11,12)$. While these approaches suggest a plausible mechanism for RA function, the results should be interpreted with caution. Manipulation of essential signaling pathways frequently leads to embryonic lethality and potential indirect effects from

\section{METHOD SUMMARY}

Here we establish a novel culture system wherein immature limb cartilage isolated from embryonic day (E13) mouse embryos underwent proper chondrogenesis following removal of nascent epidermis and incubation at an air-medium interface in a medium containing high concentrations of $\mathrm{CaCl}_{2}$, ascorbic acid, and $\beta$-glycerophosphoric acid. 
defects caused in other tissues or at earlier stages. As an alternative, an in vitro model was developed in which isolated precartilaginous cells were maintained in a microenvironment appropriate for cartilage differentiation in order to analyze the effects of RA. These experiments also provided information on the molecular mechanisms of embryonic chondrogenesis (13). However, currently available culture models have severe limitations because cartilaginous units with spatial differentiation cannot be induced from isolated chondroblasts or precartilaginous cells.

Here we established a novel culture method that duplicates chondrogenesis of an intricately shaped body part in vitro. Our system allowed us to examine the differential effects of RA on the spatial control of morphogenesis as well as on calcification of limb cartilage.

\section{Materials and methods}

Organ culture of embryonic limbs All experiments using mice were conducted following the policies and procedures of the Institutional Animal Care and Use Committee of Kwansei Gakuin University. Forelimbs of embryonic day 13 (E13) embryos were collected from pregnant ICR mice (Japan SLC Inc., Shizuoka, Japan), and the ectoderm-derived epidermal cell layer was microsurgically removed (for detailed procedure, see Supplementary Figure S1). The naked limbs containing forearms and palms were placed on a transparent porous membrane (diameter: $13 \mathrm{~mm}$, pore size: $8 \mu \mathrm{m}$; GE Healthcare, Tokyo, Japan) floating on basal medium (BM) consisting of DMEM/HamF12 medium supplemented with 10\% fetal calf serum (FCS) as previously described for the embryonic tail bud (Supplementary Figure S1) (14). To induce calcification in the explant, $10 \mathrm{mM} \mathrm{CaCl}_{2}(\mathrm{Ca}), 50 \mu \mathrm{g}$ / $\mathrm{mL}$ ascorbic acid (AA) (Invitrogen, Tokyo, Japan), and $10 \mathrm{mM} \beta$-glycerophosphoric acid (BGP), all of which reportedly support differentiation of chondrogenic or osteogenic cells (15-17), were added to the medium. To test the effect of RA, all-trans RA (Sigma-Aldrich, Tokyo, Japan) was added to the medium at a concentration of $5 \mu \mathrm{M}$ since this concentration produced the maximum effect. Presumably, lower concentrations are insufficient to supply RA to all the cells in the rudiments from the medium under the membrane. The medium was changed every 5 days, and the explants were photographed on day 5 or 10 , followed by wholemount skeletal staining. For some cultures, the naked limb was microsurgically divided into forearm and carpal/phalange, or the radius/ulna was divided into three equal sections before cultivation. For cell pellet cultures, the limbs of E13 embryos were treated with trypsin at $4^{\circ} \mathrm{C}$ overnight, dissociated in the presence of $10 \%$ serum and DNase $(10 \mu \mathrm{g} /$ $\mathrm{mL})$, and then centrifuged. The resulting chondrocyte cell pellet $(1 \mu \mathrm{l})$ was placed on a porous membrane and cultured as described for limb rudiments.

\section{Wholemount staining}

Wholemount staining of mouse embryos or cultured limbs was performed as described previously (14). In brief, samples were sequentially treated with ethanol, acetone, and ethanol containing 3\% acetic acid, $0.1 \%$ alizarin red $\mathrm{S}$, and $0.3 \%$ alcian blue. To analyze spatial calcification patterns, a staining solution containing only alizarin red was used. After dehydration with $100 \%$ ethanol, samples were incubated in a solution containing $1 \%$ potassium hydroxide and $20 \%$ glycerol for 3 days.

\section{Analysis of spatiotemporal} calcification in the limb

Calcification patterns in the limbs were analyzed using ImageJ (18). Limbs from mouse E13 or E18, or cultured explants, were stained with alizarin red and photographed. The images of radius and carpi/ phalange of the first finger were divided into 10 or 15 equal portions, respectively. The staining intensity was quantified, and the signal of the most intensely stained region was normalized to 100 , while that of an unstained region was normalized to 0. Quantifications of five independent samples for each category were plotted as line graphs.

Assessment of the effect of retinoic acid on model cell lines The precartilaginous cell line ATDC5 (American Type Culture Collection, Manassas, VA) was maintained in DMEM/ HamF12 supplemented with 5\% FCS, 1.0 $\mathrm{mg} / \mathrm{mL}$ apo-transferrin (Sigma-Aldrich) and $1.0 \times 10^{-8} \mathrm{M}$ sodium selenite (SigmaAldrich). The osteoblast cell line MC3T3-E1 (RBRC-RCB1126; RIKEN BioResource Center, Cell Engineering Division Cell Bank) was maintained in $\alpha$-MEM supplemented with 10\% FCS. To induce calcification, these cells were cultured for 2 weeks in
$\alpha$-MEM containing AA ( $50 \mu \mathrm{g} / \mathrm{mL})$ and BGP (10 mM). Additional $\mathrm{CaCl}_{2}$ was not added to the medium for induction, since this would have increased the calcium concentration to a level that is toxic to both cell types. To quantify calcification, the cells were stained with alizarin red as described for the wholemount staining, and absorbance at $570 \mathrm{~nm}$ was measured using a plate reader (Thermo Scientific, Vantaa, Finland). To analyze cell proliferation, cell numbers were evaluated using Alamar blue reagent (Invitrogen) following the manufacturer's protocol. In brief, Alamar blue reagent was added to the culture (1:40 dilution), and the absorbance ratio at 570 and $600 \mathrm{~nm}$ in the medium was measured on days 1, 3, and 7 .

Quantitative real-time PCR (qRT-PCR) Total RNA was extracted from cells cultured with or without RA for 2 weeks using an RNeasy Mini Kit (Qiagen, Tokyo, Japan). The RNA was subsequently reverse transcribed with ReverTra Ace (Toyobo, Osaka, Japan), and qRT-PCR was performed using FastStart Essential DNA Green Master on a LightCycler Nano system (Roche, Tokyo, Japan) according to the manufacturer's protocol. The signal of osteocalcin mRNA was normalized to that of $\beta$-actin. The primer pairs used in this study were: 5'-TGTTACCAACTGGGACGACA-3' and 5'CTTTTCACGGTTGGCCTTAG-3'; and 5'-AAGCAGGAGGGCAATAGGT-3' and 5'-TTTGTAGGCGGTCTTCAAGC-3; for $\beta$-actin and osteocalcin, respectively.

\section{Statistical analyses}

The results are expressed as the mean \pm SD of at least three independent experiments. Data were analyzed using MannWhitney $t$-test, and a $p$-value of $<0.05$ was considered statistically significant.

\section{Results and discussion}

Staining mouse embryos with alcian blue/ alizarin red showed that sclerotomederived chondrocytes had aligned to form uncalcified cartilage at E13 and then underwent active chondrogenesis to form complete bone templates with a clear calcification pattern by E18 (Figure 1A). To follow chondrogenesis in limb cartilage, limbs of E13 embryos were cultured at the air-medium interface after removal of the nascent epidermis, which has been shown to allow three-dimensional (3-D) chondrogenesis in the embryonic tail bud (14). Similar to what has reported for tail 
A
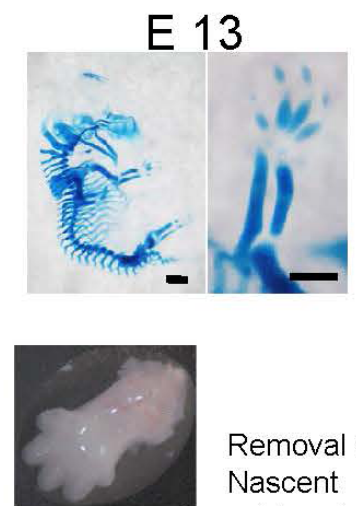

Removal of Nascent epidermis
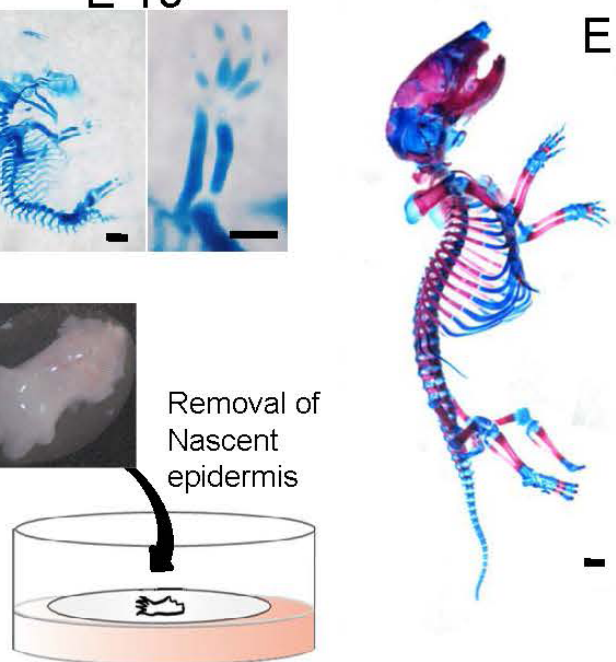

E 18
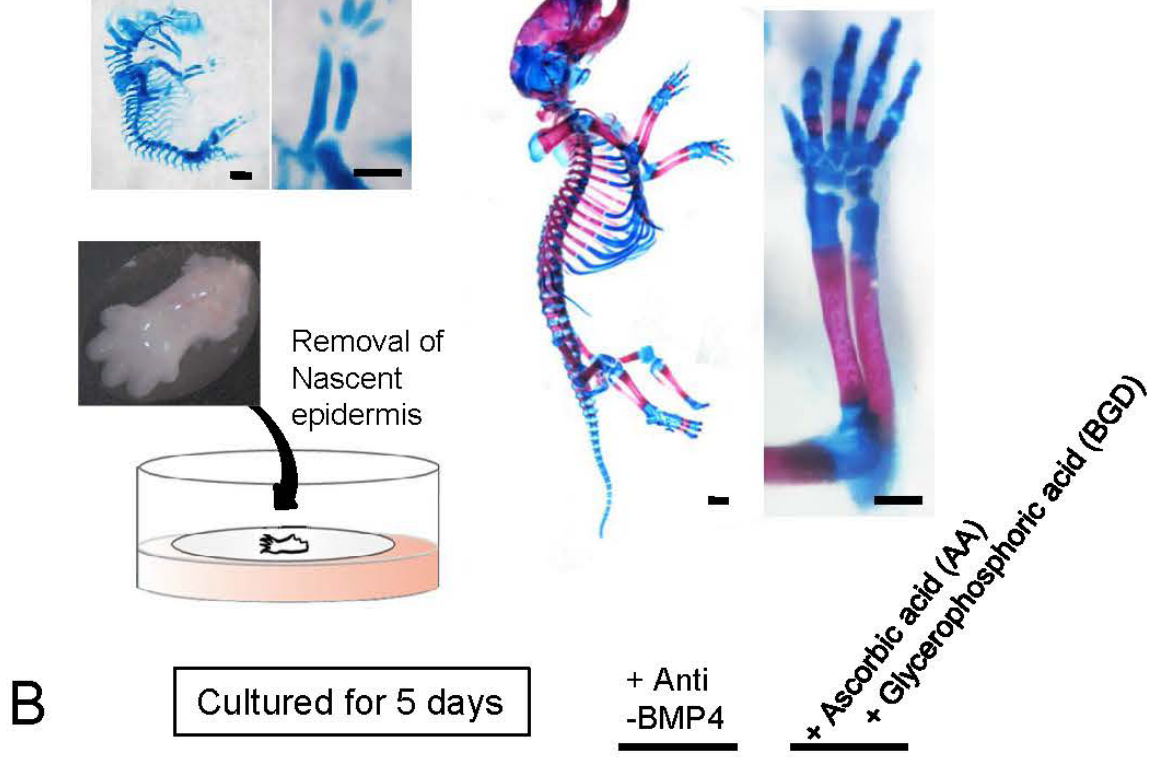

Basal meidum (BM) Intact limb
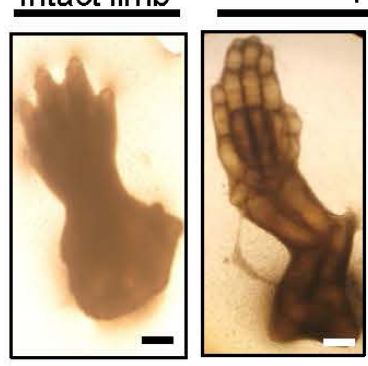

Pre-removal of epidermis
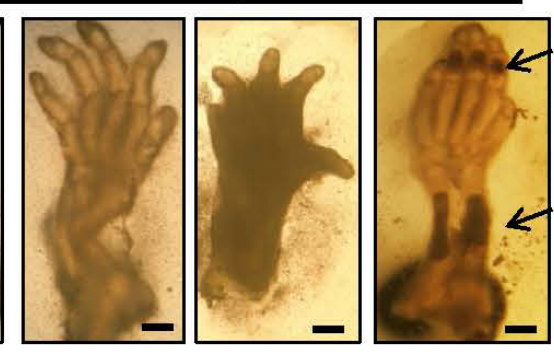

C

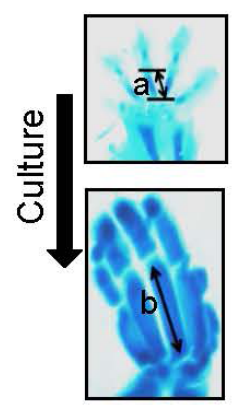

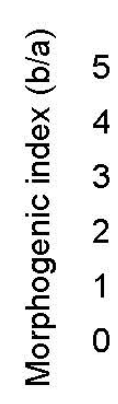

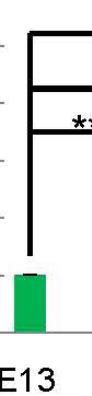

$* *$

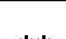
**
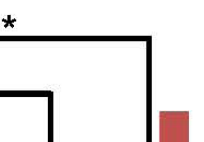

$$
{ }^{* *} \underset{n=5}{P}<0.01
$$

dayo
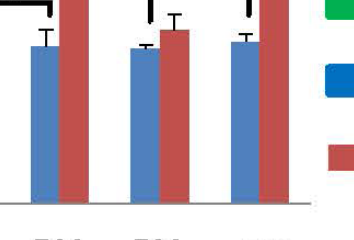

day5

day 10

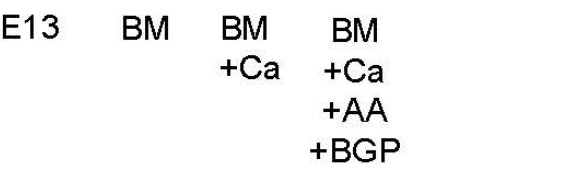

Figure 1. Development of mouse embryonic limb cartilage. (A) Whole body or limbs of an embryonic day 13 (E13) (upper left) and an E18 embryo (right) were stained with alcian blue/alizarin red. Calcifying areas were undetectable in the cartilage of E13 embryos. The culture procedure used for embryonic limb rudiments is depicted on the lower left of (A). Bars: $250 \mu \mathrm{m}$. (B) Forelimbs of E13 embryos were cultured for 5 days with [basal medium (BM) + calcium (Ca)] or without additional Ca (BM alone). In some cultures, anti-BMP antibodies $(2 \mu \mathrm{g} / \mathrm{mL})$ or ascorbic acid (AA) and $\beta$-glycerophosphoric acid (BGP) were added to the medium, which resulted in morphogenetic defects or the appearance of dark areas (arrows), respectively. Bars: $250 \mu \mathrm{m}$. (C) Growth of limb cartilage was quantified by measuring the length of metacarpal cartilage before (a) and after (b) cultivation for 5 and 10 days. The value b/a was defined as the morphogenetic index. buds, chondrocytes in the nascent forearm and palm underwent morphogenesis and formed the normal cartilaginous structures of the radius, ulna, wrist, and phalanges, even when grown in just the BM (Figure 1, $B$ and $C$ ). These phenotypic features were lost when the activity of BMP4, a factor essential for this process (19), was blocked by antibodies. This suggests that chondrogenesis was still controlled by normal developmental regulation when using this culture method (Figure 1B). On the other hand, pre-removal of epidermis appeared to be necessary for culture, because otherwise chondrocytes underwent apoptosis during cultivation (Figure 1B). Presumably, the stratifying epidermis of the intact limbs forms a barrier in ex vivo cultures (20) preventing trafficking of nutrients and waste products, thus inducing apoptosis. Intriguingly, supplementation of medium with $\mathrm{Ca}$, $A A$, and $B G P$ had no effect on the growth of each cartilage unit (Figure 1C) but did result in the appearance of dark areas that roughly overlapped with calcification areas in the limb cartilage of E18 embryos (Figure 1B). This significant change was dependent on all three supplements since elimination of any of them dramatically reduced its occurrence frequency (data not shown). Indeed, these supplements are well known to reliably support bone formation in vitro $(16,17)$.

To clarify whether nascent limbs cultured with $\mathrm{Ca}, \mathrm{AA}$, and BGP underwent proper calcification, calcified areas in explants were visualized with alizarin red. Limb cultures exhibited morphological similarities with the limb bones of later stage-embryos (e.g., E18). In addition, we observed a mosaic-like pattern and a central expansion of calcification area in the phalanges and radius/ulna, respectively. This was dependent on addition of the supplements, which further confirms that the supplement cocktail can induce calcification reliably and specifically (Figure 2, $A$ and $B$ ). Considering that the osteogenic cells that replace the cartilage template are delivered solely via blood vessels $(21,22)$ and that endothelial cells were undetectable in the cultured rudiments (data not shown), the calcified areas should be composed of mainly chondrocytes. However, we detected weak expression of collagen type I, a marker for osteoblasts instead of collagen type II, a marker for immature and mature chondrocytes (Supplementary Figure S2), suggesting 
calcified chondrocytes were removed and replaced by osteogenic cells. In addition, previous studies indicated that hypertrophic chondrocytes could undergo transdifferentiation into osteoblasts (23). Thus, it also is conceivable that osteoprogenitor cells residing in the rudiments partly participate in the regional calcification. Using this culture system, we found that in explants, hyperactivation of RA signaling severely disrupted morphogenesis and radically perturbed the calcified pattern (Figure 2, $A$ and $B$ ). In fact, hyperactivation of RA signaling has been known to inhibit chondrogenesis of mammalian limbs $(24,25)$.

Previous studies have demonstrated a substantial involvement of RA signaling in the growth and arrangement of chondrocytes, thereby affecting the regional mineralization of cartilage $(25,26)$. To investigate the relationship between the morphological defect and abnormal calcification pattern, limbs were microsurgically dissected prior to induction of chondrogenesis. While forearms and phalanges still independently underwent normal development and created the programmed calcification pattern, those treated with RA failed to undergo normal morphogenesis and showed aberrant calcification (Figure 3A). Intriguingly, we found that the RA-triggered defects in the cartilaginous structure were more prominent in forearms than in the carpi/phalanges (Figure 3A), demonstrating that RA has position-dependent effects. When the forearm portion of the limb was further dissected equally into three fragments, the overall calcification pattern was still formed in each fragment, and RA was able to induce excess calcification (Figure 3B). Notably, active calcification occurred in the central part of the forearm (llb fragment in Figure 3B) regardless of RA treatment, while growth and morphogenesis of this part was severely compromised even in the absence of the supplement cocktail for calcification (Figure 3B). These results suggest that the forelimb cartilage at the E13 embryonic stage was already endowed with a region-specific potential for morphogenesis. To further test the effect of RA on calcification in the absence of morphogenetic events, we isolated cells from the limbs of E13 embryos, thereby eliminating positional information, a critical element of morphogenesis $(27,28)$. Cell pellets were prepared by centrifugation and cultured on
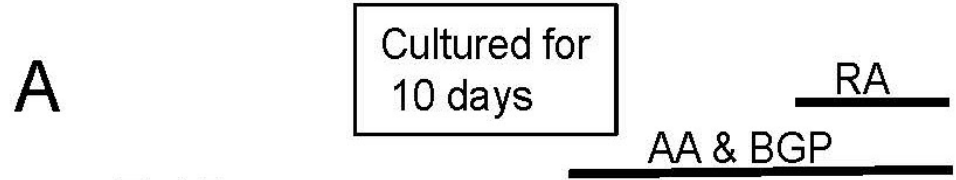

E 18
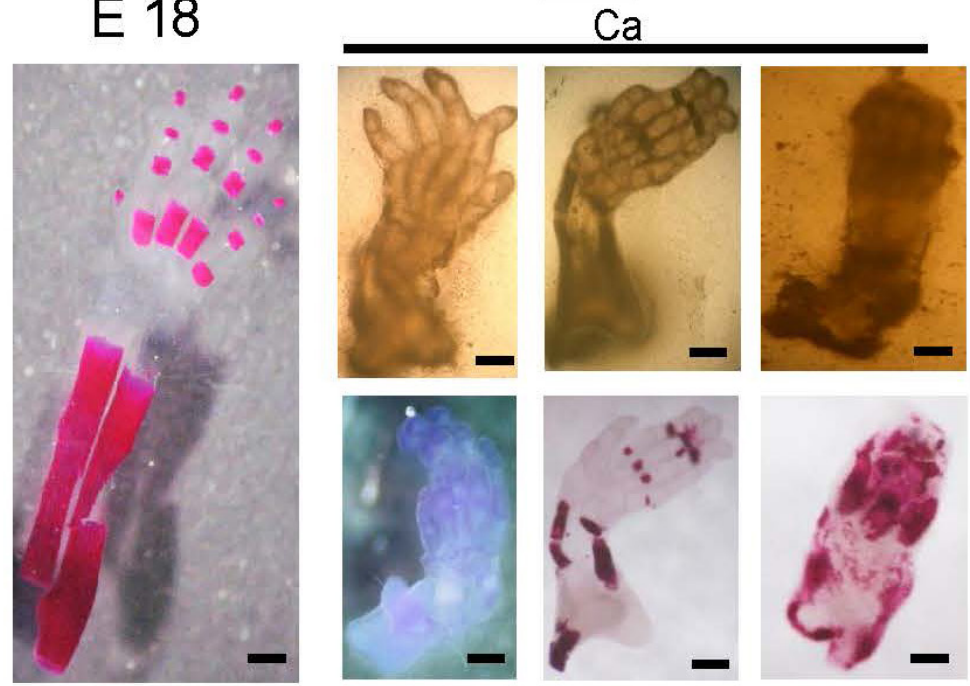
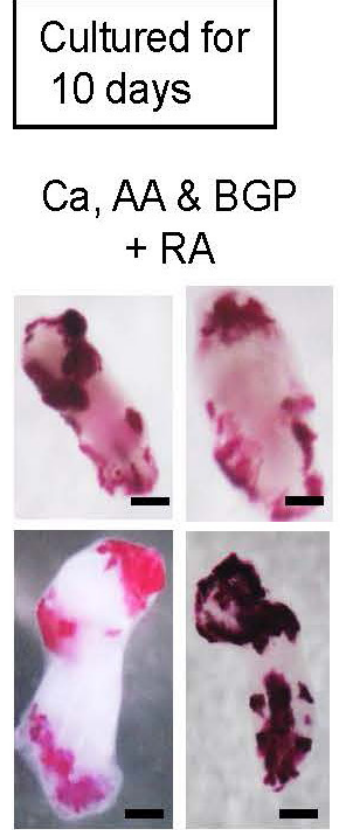

B
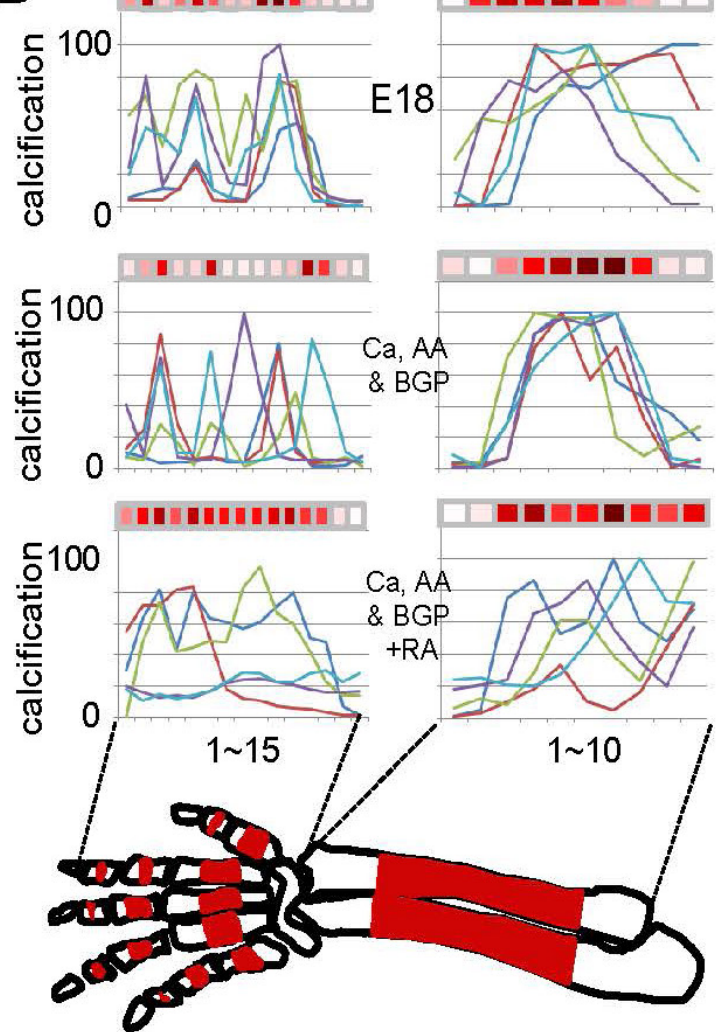

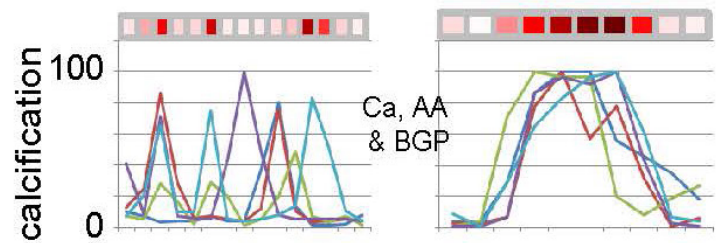

Figure 2. Calcification pattern in forelimbs cultured for 10 days. (A) Forelimbs of embryonic day (E18) embryo (left) and the explants cultured for 10 days with additional calcium (Ca), ascorbic acid (AA), and/ or $\beta$-glycerophosphoric acid (BGP) (right panels) were stained with alizarin red. In some cultures, retinoic acid (RA) was added to the medium. Right upper panel: microscopic images before staining. Lower panel: representative images of cultures with CA, AA, BGP, and RA. (B) Quantification of the calcification pattern. The staining intensity in the divided areas was quantified, and polygonal line graphs are shown for five different samples for each category. An image of a representative sample is shown above each line graph. The lines did not overlap, even in samples of E18 embryos. However, the mosaic pattern was evident in the rudiments cultured with $\mathrm{Ca}, \mathrm{AA}$, and BGP. RA dramatically perturbed the calcification program. 

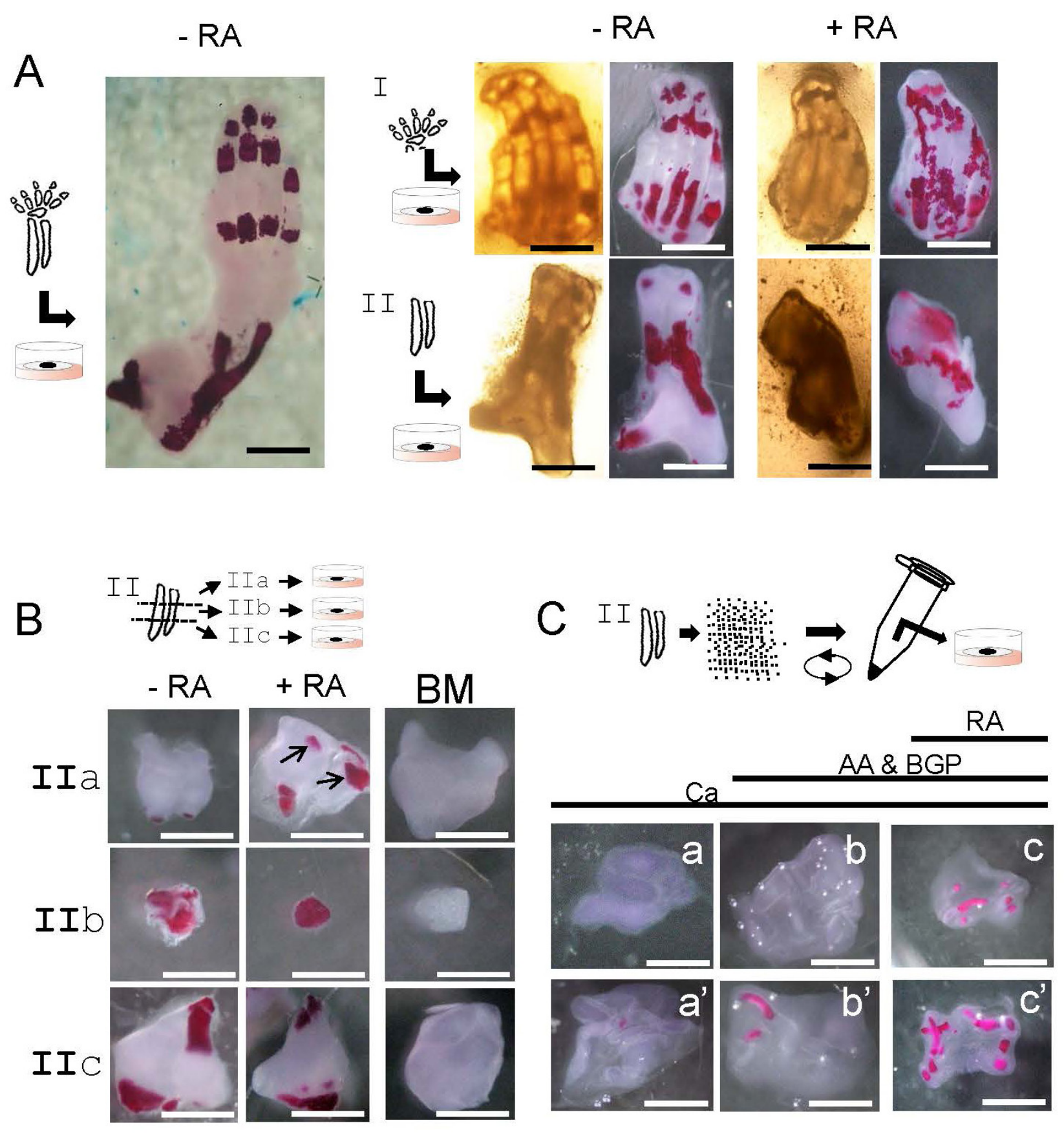

Figure 3. Morphogenesis and calcification in the forelimb fragments. (A) Carpi/phalange (I) and radius/ulna (II) were separately cultured for 10 days with medium containing alcium (Ca), ascorbic acid (AA), and $\beta$-glycerophosphoric acid (BGP) in the presence or absence of retinoic acid (+RA or -RA), followed by calcification staining with alizarin red (right panels). The left panels show the microscopic images before staining. The large image on the left shows a cultured whole forelimb for comparison. (B) The radius/ulna was dissected into three (IIa, IIb and IIc) equal sections, which were cultured separately with or without RA, followed by calcification staining with alizarin red. Arrows: Abnormal calcification in samples treated with RA. (C) Cell pellets of nascent chondrocytes were cultured with medium containing Ca, AA, and/or BGP in the presence or absence of RA, followed by staining with alizarin red. Two typical pellets for each category ( $a$ and a', b and b', c and c') are shown. Bars: 250 um.

a membrane floating on medium to provide these cells with a 3-D environment similar to that of limb rudiments. After incubation, cells in the pellets were assembled into non-directional units with regional calcification (Figure 3C), but distinct bone shapes never formed in any of the cultured cell pellets. We found that RA treatment led to an increase in calcification in the cell pellets (Figure $3 \mathrm{C}$ ), suggesting that RA-triggered calcification does not require cartilage morphogenesis. The formation of non-directional units in all of the cell pellets might be the result of segregation of cell subpopulations because the limb cartilage of E13 embryos already consists of a heterogeneous cell population in terms of morphogenesis/differentiation potential. The sorting and segregation of subpopulations in cell aggregates is known to be navigated by cell adhesion molecules $(29,30)$ or an Ephrin/Eph system (31), and an uneven cluster distribution of these molecules in the developing cartilage has 

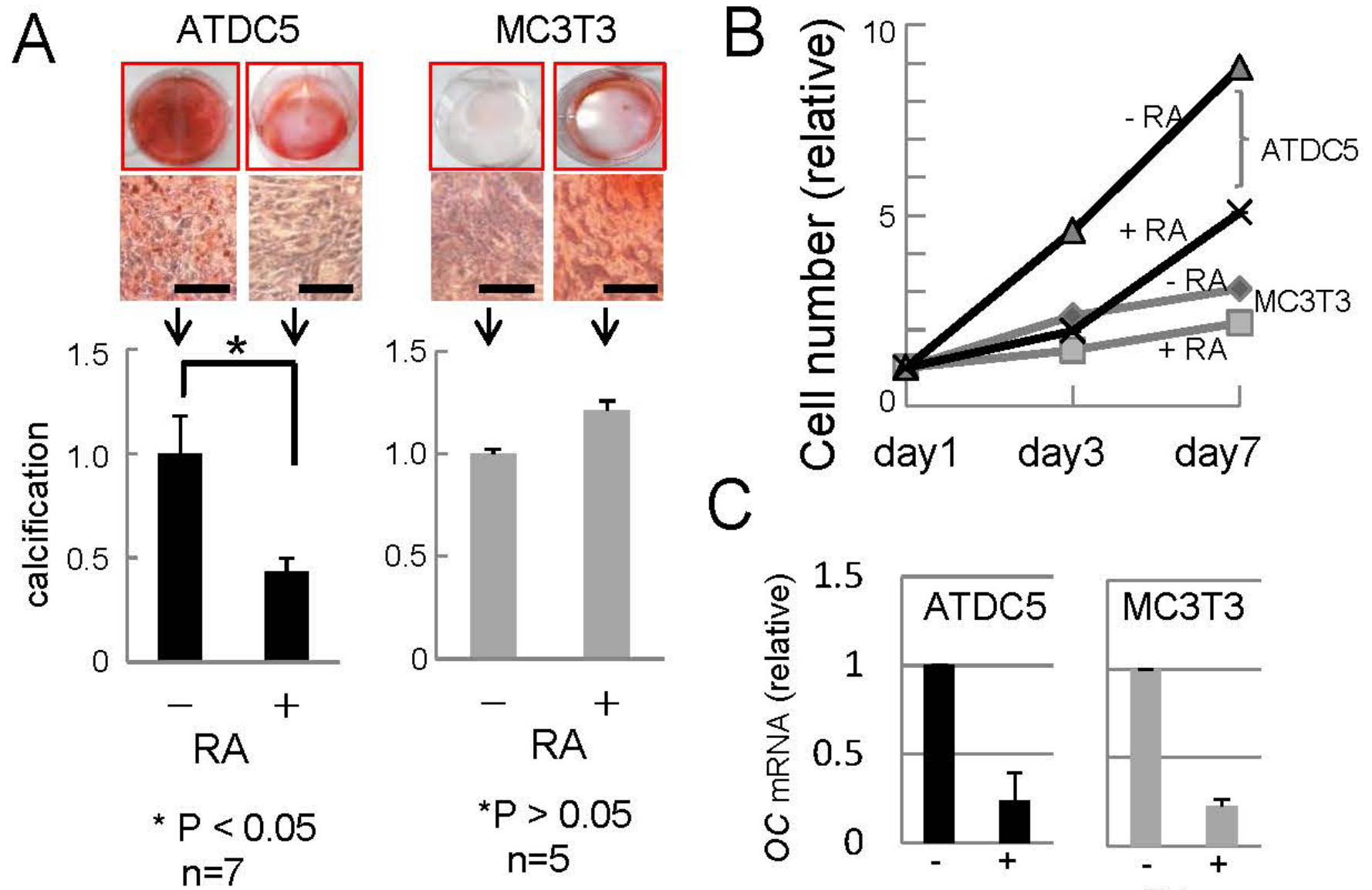

Figure 4. Effect of retinoic acid (RA) on calcification in the model cell lines. Effects of RA on calcification (A), growth (B), and expression of osteocalcin mRNA (C) in ATDC5 and MC3T3 cells cultured in the presence or absence of RA (+RA or -RA). Both cell types reached confluence before staining with alizarin red $S$, regardless of RA treatment ( $A$, upper paned), and the expression amount of $\beta$-actin was almost same (data not shown). Bars in (A): $125 \mathrm{um}$. While growth of both cell types was inhibited by RA treatment (B), the amount of osteocalcin (OC) mRNA normalized to that of $\beta$-actin was decreased (C). Thus, RA appeared to decrease differentiation in ATDC5 cells.

been reported (30). These experiments reveal that RA signaling plays independent roles in both morphogenesis and calcification, and both functions are closely related in the developmental processes for proper chondrogenesis.

Our results so far show that RA can induce cartilage calcification in ex vivo limb cultures as well as isolated chondrocytes. In contrast, previous studies using cell lines found that hyperactivation of RA signaling inhibits calcification $(32,33)$. To reconcile this difference, we tested the effect of RA on $A A$ and $B G P$-triggered calcification in two model cell lines, the precartilaginous cell line ATDC5 and the osteoblast cell line MC3T3. We were able to confirm that RA led to growth arrest and down-regulation of osteocalcin, a protein responsible for bone mineralization and calcium ion homeostasis (33), and clearly decreased calcification in ATDC5 cells (Figure 4, A-C). This effect would suggest that the cellular response to RA is affected by the absence of positional cues. However, a model cell line grown inside a plastic dish is a highly artificial system. Our novel culture system that uses reconstituted 3-D pellets lacking any positional information $(27,28)$ clearly showed that RA could induce calcification in the absence of morphogenesis. These results demonstrate the limitations of model cell lines in replicating mammalian chondrogenesis in vitro and highlight the potential of our culture method for analyses of chondrogenesis and endochondral ossification.

\section{Author contributions}

E.M. and Y.H. designed experiments, and E.M. and K.S. performed them. K.M. and Y.H. critically checked the data and suggested additional experiments to confirm them. Y.H wrote the manuscript.

\section{Acknowledgments}

We are grateful to all members of the Hirai laboratory for helpful discussions. Part of this study was supported by Grant-Aid for Scientific Research (KAKENHI 24590365).

\section{Competing interests}

The authors declare no competing interests.

\section{References}

1. Balling, R., C.F. Lau, S. Dietrich, J. Wallin, and P. Gruss. 1992. Development of the skeletal system. Ciba Found Symp. 165:132140.

2. Cancedda, R., P. Castagnola, F.D. Cancedda, B. Dozin, and R. Quarto. 2000. Developmental control of chondrogenesis and osteogenesis. Int. J. Dev. Biol. 44:707714.

3. Kawaguchi, H. 2008. Endochondral ossification signals in cartilage degradation during osteoarthritis progression in experimental mouse models. Mol. Cells 25:1-6. 
4. Ide, H. 2012. Bone pattern formation in mouse limbs after amputation at the forearm level. Dev Dyn. 241:435-441.

5. Cunningham, T.J., X. Zhao, L.L. Sandell, S.M. Evans, P.A. Trainor, and G. Duester. 2013. Antagonism between retinoic acid and fibroblast growth factor signaling during limb development. Cell Rep. 3:1503-1511.

6. Goldring, M.B., K. Tsuchimochi, and K. Ijiri. 2006. The control of chondrogenesis. J. Cell. Biochem. 97:33-44.

7. Lorda-Diez, C.I., J.A. Montero, J.A. Garcia-Porrero, and J.M. Hurle. 2014. Divergent differentiation of skeletal progenitors into cartilage and tendon: lessons from the embryonic limb. ACS Chem. Biol. 9:72-79.

8. Cunningham, T.J. and G. Duester. 2015. Mechanisms of retinoic acid signalling and its roles in organ and limb development. Nat Rev Mol Cell Biol. 16:110-123

9. Hayamizu, T.F. and S.V. Bryant. 1994. Reciprocal changes in Hox D13 and RAR-beta 2 expression in response to retinoic acid in chick limb buds. Dev. Biol. 166:123-132.

10. Lu, H.C., J.P. Revelli, L. Goering, C. Thaller, and G. Eichele. 1997. Retinoid signaling is required for the establishment of a ZPA and for the expression of Hoxb-8, a mediator of ZPA formation. Development 124:1643-1651.

11. Galdones, E., D. Lohnes, and B.F. Hales. 2006. Role of retinoic acid receptors alpha1 and gamma in the response of murine limbs to retinol in vitro. Birth Defects Res. A Clin. Mol. Teratol. 76:39-45.

12. Maden, M. 2000. The role of retinoic acid in embryonic and post-embryonic development. Proc. Nutr. Soc. 59:65-73.

13. Gigout, A., M. Jolicoeur, M. Nelea, N. Raynal, R. Farndale, and M.D. Buschmann. 2008. Chondrocyte aggregation in suspension culture is GFOGER-GPP- and beta1 integrindependent. J. Biol. Chem. 283:31522-31530.

14. Aono, Y. and Y. Hirai. 2011. A culture system for the live analysis of successive developmental processes and the morphological control of mammalian vertebral cartilage. Cytotechnology 63:269-277.

15. Cruz, A.C., M.L. Silva, T. Caon, and C.M Simoes. 2012. Addition of bone morphogenetic protein type 2 to ascorbate and $\beta$-glycerophosphate supplementation did not enhance osteogenic differentiation of human adipose-derived stem cells. J Appl Oral Sci. 20:628-635.

16. Descalzi Cancedda, F., C. Gentili, P Manduca, and R. Cancedda. 1992. Hypertrophic chondrocytes undergo further differentiation in culture. J. Cell Biol. 117:427-435

17. Fiorentini, E., D. Granchi, E. Leonardi, N. Baldini, and G. Ciapetti. 2011. Effects of osteogenic differentiation inducers on in vitro expanded adult mesenchymal

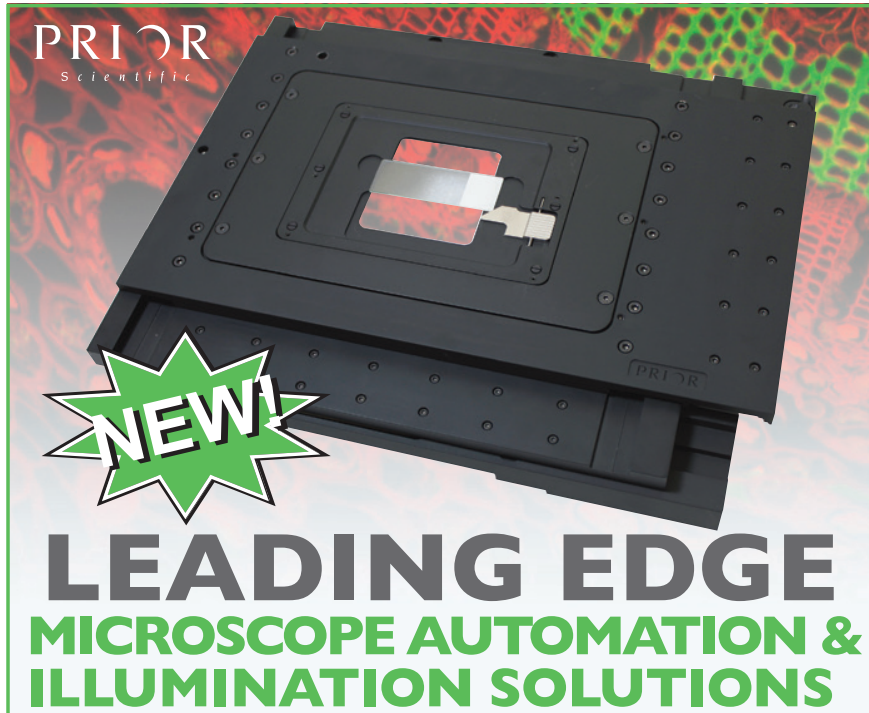

From the highest precision linear motor motorized microscope stages to the most advance fluorescence illumination systems, Prior Scientific manufactures the highest performing and most reliable microscope automation equipment available.
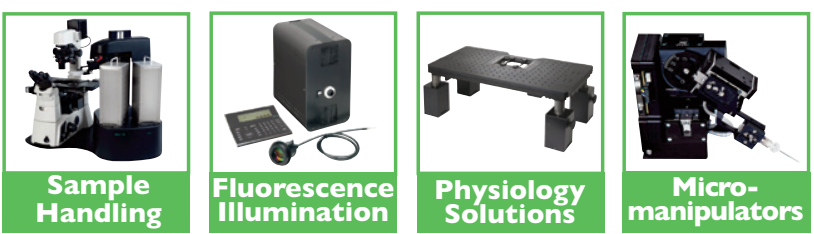

Prior Scientific, Inc. 80 Reservoir Park Dr. Rockland, MA. 02370 Tel: 800-877-2234 Web: www.prior.com stromal cells. Int. J. Artif. Organs 34:9981011.

18. Schneider, C.A., W.S. Rasband, and K.W. Eliceiri. 2012. NIH Image to ImageJ: 25 years of image analysis. Nat. Methods 9:671-675.

19. Chen, D., M. Zhao, and G.R. Mundy. 2004. Bone morphogenetic proteins. Growth Factors 22:233-241.

20. Hirai, Y., A. Nose, S. Kobayashi, and M. Takeichi. 1989. Expression and role of E- and P-cadherin adhesion molecules in embryonic histogenesis. I. Lung epithelial morphogenesis. Development 105:263-270.

21. Ashraf, S. and D.A. Walsh. 2008. Angiogenesis in osteoarthritis. Curr. Opin. Rheumatol. 20:573-580.

22. Maes, C. 2013. Role and regulation of vascularization processes in endochondral bones. Calcif. Tissue Int. 92:307-323.

23. Cancedda, R., F. Descalzi Cancedda, and P. Castagnola. 1995. Chondrocyte differentiation. Int. Rev. Cytol. 159:265-358.

24. Bryant, S.V. and D.M. Gardiner. 1992 Retinoic acid, local cell-cell interactions, and pattern formation in vertebrate limbs. Dev. Biol. 152:1-25.

25. Underhill, T.M. and A.D. Weston. 1998 Retinoids and their receptors in skeletal development. Microsc. Res. Tech. 43:137155.

26. Lee, G.S., D.M. Kochhar, and M.D. Collins. 2004. Retinoid-induced limb malformations. Curr. Pharm. Des. 10:2657-2699.

27. Wolpert, L. 1971. Positional information and pattern formation. Curr. Top. Dev. Biol. 6:183-224

28. Wolpert, L. 1994. Positional information and pattern formation in development. Dev. Genet. 15:485-490.

29. Oberlender, S.A. and R.S. Tuan. 1994 Expression and functional involvement of $\mathrm{N}$-cadherin in embryonic limb chondrogenesis. Development 120:177-187.

30. Woods, A., G. Wang, and F. Beier. 2007 Regulation of chondrocyte differentiation by the actin cytoskeleton and adhesive interactions. J. Cell. Physiol. 213:1-8.

31. Compagni, A., M. Logan, R. Klein, and R.H. Adams. 2003. Control of skeletal patterning by ephrinB1-EphB interactions. Dev. Cell 5:217-230.

32. Cohen-Tanugi, A. and N. Forest. 1998 Retinoic acid suppresses the osteogenic differentiation capacity of murine osteoblastlike 3/A/1D-1M cell cultures. Differentiation 63:115-123

33. Kirimoto, A., Y. Takagi, K. Ohya, and H. Shimokawa. 2005. Effects of retinoic acid on the differentiation of chondrogenic progenitor cells, ATDC5. J. Med. Dent. Sci. 52:153-162.

Received26January2015; accepted25March2015

Address correspondence to Yohei Hirai, Department of Bioscience, Kwansei Gakuin University, Sanda, Japan. E-mail: y-hirai@kwansei.ac.jp

To purchase reprints of this article, contact: biotechniques@fosterprinting.com 